\title{
Psoas Major Muscle
}

National Cancer Institute

\section{Source}

National Cancer Institute. Psoas Major Muscle. NCI Thesaurus. Code C33420.

A striated muscle located in the iliac region that originates both on the transverse processes of the twelfth thoracic through fifth lumbar vertebrae and the lateral aspects of the discs between them, and inserts on the lesser trochanter of the femur; it functions to flex the hip joint. 